\title{
TAM: A method for enrichment and depletion analysis of a microRNA category in a list of microRNAs
}

\author{
Ming Lu', Bing Shi ${ }^{1,2}$, Juan Wang ${ }^{1}$, Qun Cao ${ }^{1}$, Qinghua Cui ${ }^{*}$
}

\begin{abstract}
Background: MicroRNAs (miRNAs) are a class of important gene regulators. The number of identified miRNAs has been increasing dramatically in recent years. An emerging major challenge is the interpretation of the genomescale miRNA datasets, including those derived from microarray and deep-sequencing. It is interesting and important to know the common rules or patterns behind a list of miRNAs, (i.e. the deregulated miRNAs resulted from an experiment of miRNA microarray or deep-sequencing).

Results: For the above purpose, this study presents a method and develops a tool (TAM) for annotations of meaningful human miRNAs categories. We first integrated miRNAs into various meaningful categories according to prior knowledge, such as miRNA family, miRNA cluster, miRNA function, miRNA associated diseases, and tissue specificity. Using TAM, given lists of miRNAs can be rapidly annotated and summarized according to the integrated miRNA categorical data. Moreover, given a list of miRNAs, TAM can be used to predict novel related miRNAs. Finally, we confirmed the usefulness and reliability of TAM by applying it to deregulated miRNAs in acute myocardial infarction (AMI) from two independent experiments.

Conclusion: TAM can efficiently identify meaningful categories for given miRNAs. In addition, TAM can be used to identify novel miRNA biomarkers. TAM tool, source codes, and miRNA category data are freely available at http:// cmbi.bjmu.edu.cn/tam.
\end{abstract}

\section{Background}

MicroRNAs (miRNAs) are one class of newly identified important cellular components [1]. At the posttranscriptional level, miRNAs normally act as negative gene regulators by binding to the 3'UTR of target mRNAs through base pairing, which results in the cleavage of target mRNAs or translation inhibition [1]. Increasing evidences suggest that miRNAs play crucial roles in nearly all important biological processes, including cell growth, proliferation, differentiation, development, and apoptosis [2], and that miRNA dysfunctions are associated with various diseases [3]. Since their discovery, the number of identified miRNAs has been increasing dramatically and various high-throughput techniques related to miRNAs are continuously being developed.

\footnotetext{
* Correspondence: cuiqinghua@hsc.pku.edu.cn

'Department of Biomedical Informatics, Peking University Health Science Center, Beijing, 100191, China

Full list of author information is available at the end of the article
}

Microarrays, for example, generate experimental data at rates that exceed knowledge growth. To mine meaningful information of miRNAs, a number of tools and databases have been presented [4-12]. Among these resources, the tools for searching for the gene sets (i.e. KEGG pathways and Gene Ontology) that may be affected by one or multiple miRNAs represent some of the most important tools in miRNA bioinformatics $[6,10,11]$. A common point of these methods is that they obtain the meaningful gene sets by enrichment analysis of the in-silico predicted miRNA targets. The first limitation of these methods is the high false positives and high false negatives of the predicted miRNA targets [13]. The second limitation of these methods is that they perform analysis based on target genes and only focus on significantly enriched gene sets and therefore may fail to find some functions or biological processes associated with the inputted miRNAs. For example, miR-18a is known to be related to apoptosis
C Biomed Central

(c) 2010 Lu et al; licensee BioMed Central Ltd. This is an Open Access article distributed under the terms of the Creative Commons Attribution License (http://creativecommons.org/licenses/by/2.0), which permits unrestricted use, distribution, and reproduction in any medium, provided the original work is properly cited. 
[14], but these methods fail to find the pathway "apoptosis" for miR-18a. Finally, it seems difficult for those methods to find novel miRNAs that are related to the inputted miRNAs. Therefore, for a list of miRNAs, for example the upregulated and/or downregulated miRNAs from a miRNA microarray experiment, novel methods are needed to find the patterns behind these miRNAs.

Most of the current tools for miRNA functional annotation are based on predicted miRNA targets, mainly, because of the lack of miRNA knowledge resources. However, functional resources for protein-coding genes are easily available. Therefore, for protein-coding genes, a large number of programs for the annotation of lists of genes have been developed [15] because various gene resources such as the Kyoto Encyclopedia of Genes and Genomes (KEGG) pathway http://www.genome.jp/kegg/ and the Online Mendelian Inheritance in Man (OMIM) compendium http://www.ncbi.nlm.nih.gov/omim/ are available for protein-coding genes. Developing miRNA annotation tools should become more feasible as meaningful miRNA resources are collected. In this study, TAM, a web-accessible program for this purpose is presented. In TAM, miRNAs are integrated into different categories according to the miRNA family, genome locations, functions, associated diseases, and tissue specificity. TAM then evaluates the statistical significance (i.e., overrepresentation or underrepresentation) of each miRNA category among lists of miRNAs using the hypergeometric test. TAM is also able to search for novel miRNAs related to a given list of miRNAs. Finally, we applied TAM to the upregulated miRNAs and downregulated miRNAs in acute myocardial infarction (AMI). As expected, different meaningful miRNA categories have been identified for upregulated and downregulated miRNAs, respectively. This suggested that TAM could be an efficient method and tool for the annotation of meaningful miRNA categories for a list of miRNAs. TAM represents an alternative tool for the processing of outputs of high throughput miRNA experiments.

\section{Results and Discussion miRNA categories}

In total, we collected 257 miRNA categories according to various classification schemes, such as miRNA family, miRNA cluster, miRNA function, miRNA associated disease, and miRNA tissue specificity (see Materials and Methods). miRNAs that have common characters in any classification scheme will be integrated into one category. Figure 1 shows the detailed flowchart for the miRNA category integration procedure (Figure 1). Among the 257 miRNA categories, 58 belongs to miRNA family category (Family), 72 belongs to miRNA cluster category (Cluster), 24 belongs to miRNA function category (Function), 97 belongs to human miRNA associated disease category (HMDD), and 6 belongs to tissue specificity category (TissueSpecific) (Figure 2). These miRNA categories include more than 400 distinct miRNAs.

\section{The procedure of TAM analysis}

TAM works in four steps, as shown in Figure 3. In Step 1, a given list of miRNA for analysis is entered. In Step 2, another list of miRNA is entered as background. This step is optional; if a background list is not provided, TAM will use all miRNAs included in the miRNA database as the default background list. In Step 3, the user indicates what analysis (overrepresentation or underrepresentation) is to be performed: overrepresentation or underrepresentation. In Step 4, a result page is generated after the data is submitted. TAM evaluates the significance of each miRNA category for the given miRNAs. The miRNA categories are clustered into five classes including miRNA family, miRNA cluster, miRNA function, miRNA associated disease, and miRNA tissue specificity (Table 1). In the result page, the miRNA category, number of input miRNAs matched this category, percentage of matched miRNAs, fold of the overpresentation or underrepresentation, $\mathrm{p}$ value, Bonferroni value, and FDR value are listed, respectively. Other related miRNAs with the given miRNAs in one miRNA category will be shown when the mouse move to corresponding miRNA category.

\section{The upregulated and downregulated miRNAs in acute myocardial infarction (AMI) show different TAM annotations}

We first applied TAM to the 16 deregulated miRNA genes from a miRNA microarray experiment (Table 2), in which we previously identified 16 deregulated miRNAs ( 8 are upregulated in AMI and 8 are downregulated in AMI) in the myocardium tissue of rats with AMI and normal rats [16]. This dataset includes miRNA expression profiles across four time points (the control, 3 day, 7 day, and 14 day), each time point has three samples and each sample has two replicates. In order to investigate the meaningful rules behind these deregulated miRNAs, we identified the enriched miRNA categories for the upregulated miRNAs and downregulated miRNAs, respectively. As a result, the upregulated miRNAs and downregulated miRNAs show obviously different and even opposite enriched miRNA categories. Figure 4 shows the fold of enrichment for the most enriched miRNA categories $(P<0.01)$. Significantly, the upregulated miRNAs are enriched in miR-199a cluster $\left(\mathrm{P}=1.49 \times 10^{-4}\right)$, whereas the downregulated miRNAs are enriched in miR-181c cluster $\left(\mathrm{P}=2.71 \times 10^{-3}\right)$. For the miRNA family, the upregulated miRNAs and downregulated miRNAs are enriched in miR-17 family $\left(\mathrm{P}=4.03 \times 10^{-3}\right)$ and miR-181 family $\left(\mathrm{P}=1.64 \times 10^{-3}\right)$, respectively. For the miRNA function, the two lists of 


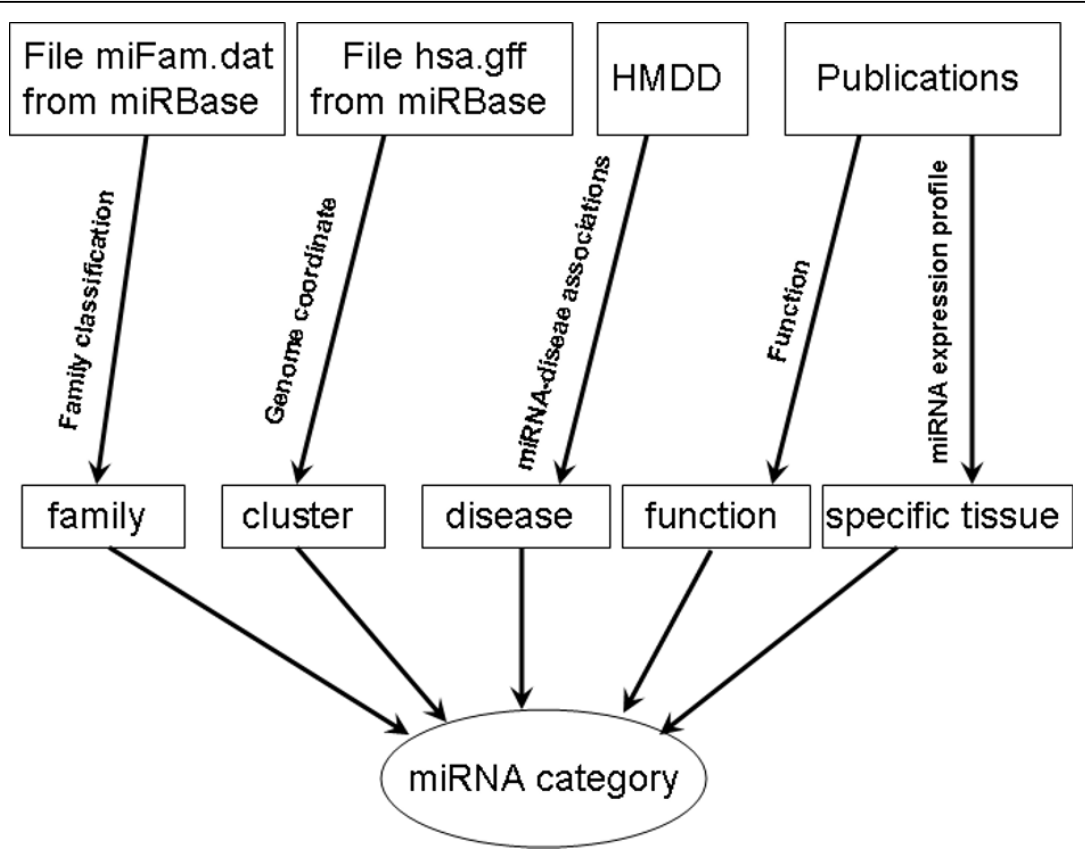

Figure 1 Classification schemes of miRNA categories. We integrated miRNAs into various categories according to five classification schemes. They are miRNA family, miRNA cluster, miRNA associated disease, miRNA function, and miRNA tissue specificity. The data sources used to generate the above miRNA categories are also given.

miRNAs show opposite functions. The upregulated miRNAs are enriched in oncogenic function $\left(\mathrm{P}=2.56 \times 10^{-4}\right)$ and immune system function $\left(\mathrm{P}=1.01 \times 10^{-3}\right)$, whereas the downregulated miRNAs are enriched in tumor suppressor function $\left(\mathrm{P}=1.36 \times 10^{-4}\right)$. Consequently, both lists of miRNAs are enriched in tumors (Table 2). In addition, the upregulated miRNAs are enriched in hypertrophic

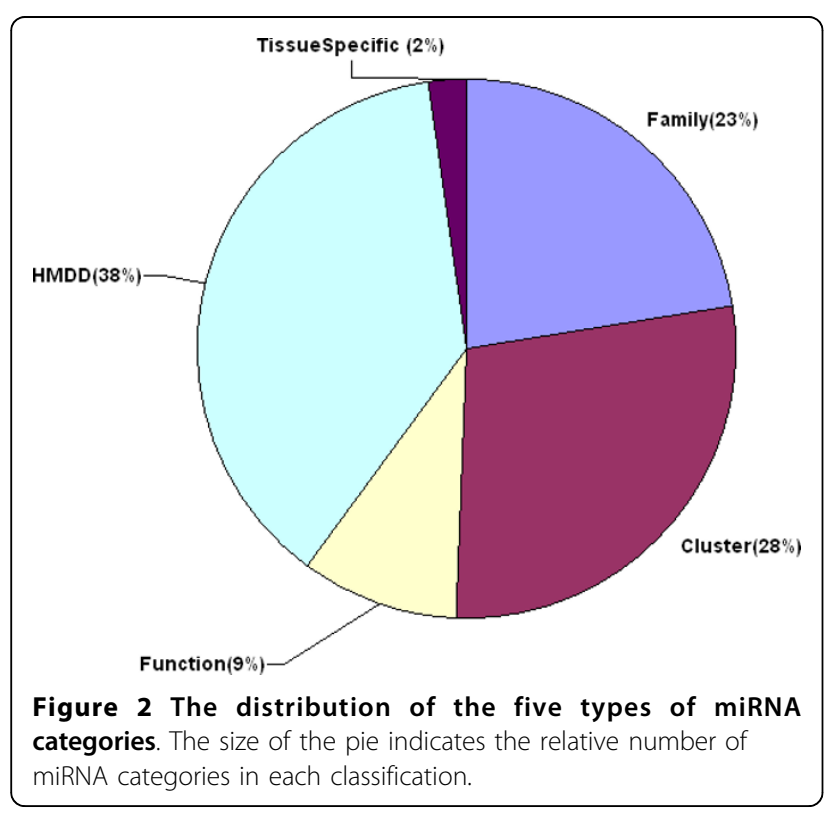

cardiomyopathy and atrophic muscular disorders, whereas the downregulated miRNAs are enriched in cardiac arrhythmias, cardiomegaly, coronary artery disease, and polycythemia vera (Table 2). In function, the upregulated miRNAs are also enriched in Akt pathway, cell cycle, HIV latency, hormones regulation, stem cell regulation, immune, and inflammation; the downregulated miRNAs are also enriched in cardiogenesis, hormones regulation, and muscle development. Finally, although not so significant, the downregulated miRNAs tend to be enriched in function of muscle development $(\mathrm{P}=0.01)$ and tend to be heart and muscle specific $(P=0.15)$. The enriched miRNA categories of AMI upregulated and downregulated miRNAs might provide help in understanding AMI. For example, the upregulated miRNAs are enriched in function of oncogenes, whereas the downregulated miRNAs are enriched in function of tumor suppressors. This result suggests that the deregulated miRNAs tend to stimulate the proliferation of cardiac fibroblasts, which is further helpful for collagen synthesis and cardiac remodeling. This may be a compensatory mechanism for acutely infracted myocardium.

To valid our method, we applied TAM to the deregulated miRNAs of AMI from another independent miRNA expression profiling experiment of AMI rat model by Rooij et al.[17]. In their study, Rooij et al. identified 39 upregulated miRNAs and 46 downregulated miRNAs, respectively. As a result, although the deregulated 


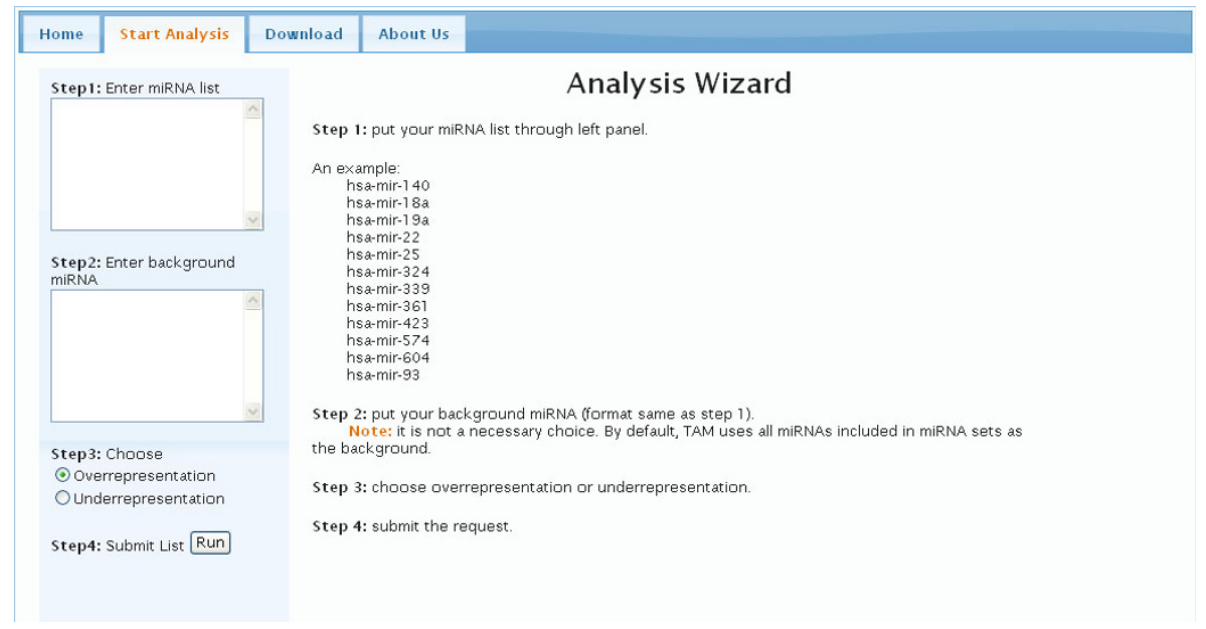

Figure 3 Analysis flowchart of TAM

miRNAs from Rooij et al.' experiment seem quite different from those of Shi et al.', the enriched miRNA categories identified by TAM have a good consistency across these two independent experiments. For example, the upregulated miRNAs from Rooij et al.' experiment are also enriched in miR-199a cluster $\left(\mathrm{P}=4.33 \times 10^{-3}\right)$, miR199 family $\left(\mathrm{P}=4.33 \times 10^{-3}\right)$, cell cycle $\left(\mathrm{P}=6.37 \times 10^{-3}\right)$, stem cell regulation $\left(\mathrm{P}=1.82 \times 10^{-6}\right)$, inflammation $\left(\mathrm{P}=3.14 \times 10^{-3}\right)$, and onco-miRNAs $\left(\mathrm{P}=5.73 \times 10^{-5}\right)$. For HMDD category, the upregulated miRNAs are also enriched in various cancer, hypertrophic cardiomyopathy $(\mathrm{P}=0.04)$ and atrophic muscular disorders $(\mathrm{P}=4.54 \times$ $\left.10^{-12}\right)$; the downregulated miRNAs from Rooij et al.' experiment are also enriched in miR-29a cluster $\left(\mathrm{P}=7.37 \times 10^{-3}\right)$, miR-29b cluster $\left(\mathrm{P}=7.37 \times 10^{-3}\right)$, hormones regulation $\left(\mathrm{P}=2.14 \times 10^{-7}\right)$, miRNA tumor suppressor $\left(\mathrm{P}=9.23 \times 10^{-3}\right)$. For HMDD category, the downregulated miRNAs are also enriched in various cancer, and polycythemia vera $\left(\mathrm{P}=7.01 \times 10^{-3}\right)$.

\section{Prediction of novel miRNAs related to AMI}

As discussed previously, one of the limitations of targetbased pathway enrichment analysis of miRNAs is that it can not predict novel miRNAs related to the inputted miRNAs. For TAM, it is very easy to perform this kind of analysis because TAM integrated miRNAs directly but not integrated miRNAs through miRNA targets. In the enriched miRNA category, the other miRNAs that are not included in the input miRNA list could be potential novel miRNAs related to the inputted miRNAs. For example, TAM analysis showed that the 16 deregulated miRNAs in AMI from Shi et al.'s study are enriched in the function of muscle development $(\mathrm{P}=0.04)$. Among the 11 miRNAs in this category, two (miR-1 and miR-499) are included in the 16 inputted miRNAs. The other 9 miRNAs (miR-24, miR-124, miR133a, miR-23a, miR-133b, miR-206, miR-221, miR-222, and $\mathrm{miR}-208 \mathrm{~b})$ in this category are predicted to be potential novel AMI related miRNAs. We confirmed four (miR-24, miR-133a, miR-221, and miR-222) of the nine miRNAs (44.4\%) are related to AMI based on the deregulated miRNAs from another independent study by Rooij et al.[17]. The results indicate that TAM is a highly reliable tool for predicting novel miRNAs that are related to inputted miRNAs.

\section{Discussion}

As the rapid development of high-throughput biological techniques, it is increasingly important to mine meaningful patterns for a given list of miRNAs. As described above, TAM represents one important tool for this purpose. Unlike tools based on in-silico predicted miRNA targets, TAM integrated miRNAs into groups directly based on miRNA annotations. Therefore, TAM represents a new

Table 1 Options provided by the TAM tool

\begin{tabular}{ll}
\hline Annotation & Description \\
\hline miRNA family & miRNA categories integrated according to miRNA conservation \\
miRNA cluster & miRNA categories integrated according to miRNA genome location \\
miRNA function & miRNA categories integrated according to their function \\
miRNA associated disease & miRNA categories integrated according to the associated disease \\
miRNA tissue specificity & miRNA categories integrated according to their tissue specificity \\
\hline
\end{tabular}


Table 2 Significant miRNA categories of upregulated and downregulated miRNAs in AMI obtained by TAM

\begin{tabular}{lcc}
\hline $\begin{array}{l}\text { miRNA } \\
\text { category }\end{array}$ & Upregulated miRNAs & Downregulated miRNAs \\
\hline Family & (miR-31; miR-18a;miR-18b; miR-214;miR-223;miR-923; miR-711;miR-199a) & (miR-499;miR-29b;miR-126; miR-1;miR-181d;miR-181c; \\
miR-451;miR-26b)
\end{tabular}

class of methods for the above purpose and represents an alternative tool for the annotations of a given list of miRNAs. Furthermore, TAM is able to predict novel miRNAs that are related to the inputted miRNAs. This enables users to find novel miRNA biomarkers for their experiments. In addition, TAM is highly dependent on the data of integrated miRNA sets and will be improved greatly when more miRNA annotation data becomes available in the future.

\section{Conclusions}

In this study, we presented a method to identify overrepresented and/or underrepresented miRNA categories

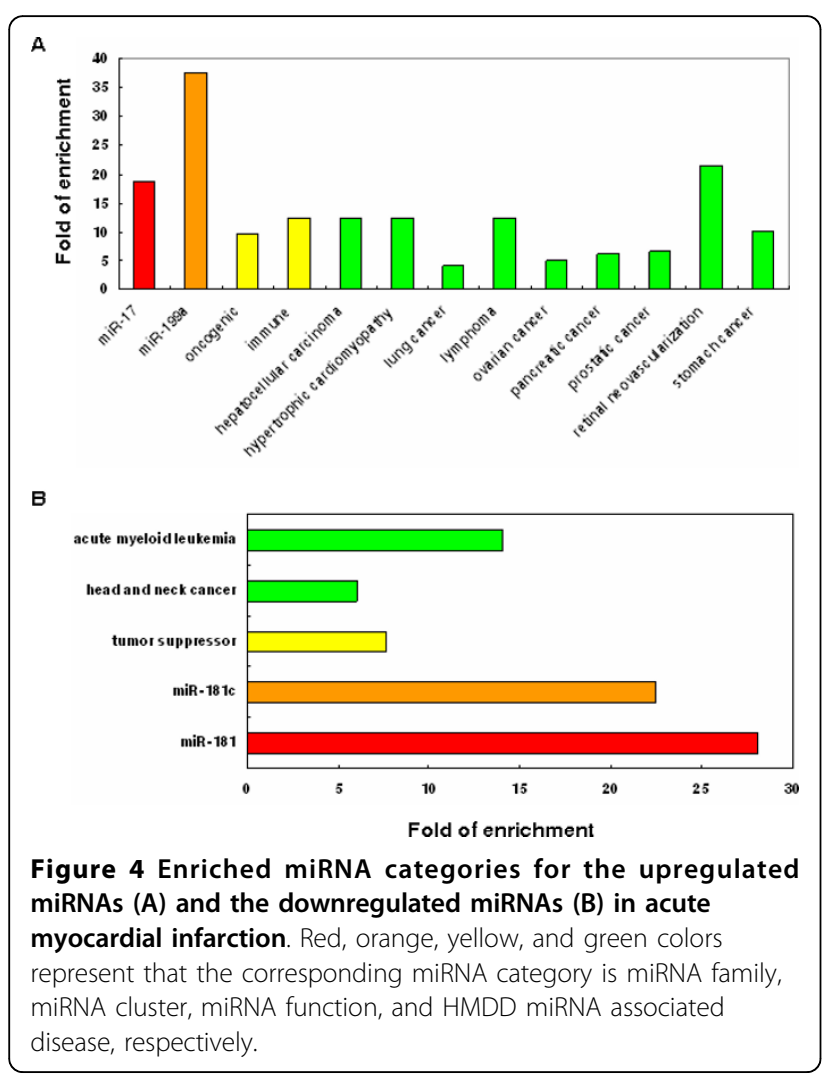

for a given list of miRNAs. Moreover, an online tool, TAM, for annotations of human miRNAs based on various miRNA sets is developed. After applying TAM to deregulated miRNAs in AMI, we show that the upregulated miRNAs and the downregulated miRNAs in AMI are enriched in different and even opposite miRNA categories, which is helpful for the understanding of AMI. In addition, TAM can be used to predict novel miRNAs that are mostly related to the input miRNAs. TAM is useful for providing potential clues for miRNAs of interest. Furthermore, TAM is scalable and will grow and improve as more miRNA resources become available. In addition, TAM can be easily reconfigured for use with other species.

\section{Methods}

miRNA sets

miRNA sets are defined as groups of miRNAs that have meaningful relationships. If any two miRNAs have meaningful relationships, for example they are associated with the same diseases, they are then integrated into one miRNA set. Here, miRNA sets were collected according to miRNA family, genome locations, function, associated diseases, and tissue specificity. Studies have indicated that miRNAs in one family are most likely derived from duplications of common ancestor miRNAs $[18,19]$, and tend to act together in various functional processes $[20,21]$. Therefore, miRNAs in one family can be considered as one miRNA set. The miRNA family data from the miRBase database was downloaded [7] and utilized in this study.

miRNAs are not located randomly in the genome but tend to exist in clusters [22]. MiRNAs in a cluster are likely to be co-transcribed and have similar expression patterns [23]. Therefore, these clustered miRNAs may be involved in similar biological processes. In this study, miRNA clusters were identified by grouping miRNAs that were within a distance of $50 \mathrm{~kb}$ in the chromosomes, according to the observation of Baskerville and Bartel [23]. The integrated miRNAs were also manually 
integrated into different sets according to their functions, as reported in publications. For example, miRNAs that were associated with the immune system were collected from a recent review paper published in Cell [24]. The miRNA sets were generated by miRNA-associated diseases based on the Human MicroRNA Disease Database (HMDD, http://cmbi.bjmu.edu.cn/hmdd), a database for miRNA disease associations [3]. The tissuespecific index values of miRNA were obtained from the study of Lu et al.[3], and tissue-specific miRNA sets were generated by collecting miRNAs with tissue specificity index values of greater than or equal to 0.7. Finally, according to the methods described above, 257 miRNA sets were generated. These miRNA sets are available for download at the TAM website.

\section{Evaluation of statistical significance}

The hypergeometric test [25], was used to determine the significant overrepresentation and/or underrepresentation of the miRNA sets among a list of miRNAs of interest. Assuming that $P$ represents the number of miRNAs included in all miRNA sets, $S$ represents the number of miRNAs included in miRNA set $A, H P$ represents the number of input miRNAs included in $P$, and $H S$ represents the number of miRNAs that are of interest included in $S$, the probability of $H S$ miRNAs of interest in miRNA set $A$ is

$$
P(x=H S)=\frac{C_{H P}^{H S} \times C_{P-H P}^{S-H S}}{C_{P}^{S}}
$$

where the symbol "C" means the combination operation. Therefore, the statistical significance of miRNA set $A$ among the miRNAs of interest are represented by Formula (2) and (3):

$$
\begin{aligned}
& P(\text { overrepresentation })=\sum_{h=H S}^{S} P(x=h) \\
& P(\text { underrepresentation })=\sum_{h=0}^{H S} P(x=h)
\end{aligned}
$$

Finally, the $\mathrm{P}$ values for all miRNA sets are adjusted by Bonferroni and FDR corrections.

\section{Availability and requirements}

Project name: TAM.

Project home page: http://cmbi.bjmu.edu.cn/tam.

Operating system: Platform independent.

Programming language: Python.
Other requirements: Apache 1.22, Jquery, Extjs, and Django.

License: GPL v3.

\section{Acknowledgements}

This work was supported by the Natural Science Foundation of China (Grant No. 30900829) and was supported by Doctoral Fund of Ministry of Education of China (Grant No. 20090001120040).

\section{Author details}

'Department of Biomedical Informatics, Peking University Health Science Center, Beijing, 100191, China. ${ }^{2}$ Department of Cardiology, Beijing Military General Hospital, Beijing, 100700, China.

\section{Authors' contributions}

QC designed this study and wrote the manuscript. ML implemented the algorithms and created the web server. BS and ML analyzed the deregulated miRNAs of AMI. JW and QC curated the HMDD miRNA categories. All authors have read and approved the final manuscript.

Received: 11 March 2010 Accepted: 9 August 2010

Published: 9 August 2010

\section{References}

1. Bartel DP: MicroRNAs: genomics, biogenesis, mechanism, and function. Cell 2004, 116(2):281-297.

2. Esquela-Kerscher A, Slack FJ: Oncomirs - microRNAs with a role in cancer. Nat Rev Cancer 2006, 6(4):259-269.

3. Lu M, Zhang Q, Deng M, Miao J, Guo Y, Gao W, Cui Q: An analysis of human microRNA and disease associations. PLOS ONE 2008, 3(10):e3420.

4. Alexiou P, Maragkakis M, Papadopoulos GL, Simmosis VA, Zhang L, Hatzigeorgiou AG: The DIANA-mirExTra web server: from gene expression data to microRNA function. PLoS One 5(2):e9171.

5. Alexiou P, Vergoulis T, Gleditzsch M, Prekas G, Dalamagas T, Megraw M, Grosse I, Sellis T, Hatzigeorgiou AG: miRGen 2.0: a database of microRNA genomic information and regulation. Nucleic Acids Res, 38 Database: D137-141.

6. Backes C, Meese E, Lenhof HP, Keller A: A dictionary on microRNAs and their putative target pathways. Nucleic Acids Res 2010, 38(13):4476-4486.

7. Griffiths-Jones S: The microRNA Registry. Nucleic Acids Res 2004, , 32 Database: D109-111.

8. Maragkakis M, Alexiou P, Papadopoulos GL, Reczko M, Dalamagas T, Giannopoulos G, Goumas G, Koukis E, Kourtis K, Simossis VA, et al: Accurate microRNA target prediction correlates with protein repression levels. BMC Bioinformatics 2009, 10:295.

9. Maragkakis M, Reczko M, Simossis VA, Alexiou P, Papadopoulos GL, Dalamagas T, Giannopoulos G, Goumas G, Koukis E, Kourtis K, et al: DIANAmicroT web server: elucidating microRNA functions through target prediction. Nucleic Acids Res 2009, , 37 Web Server: W273-276.

10. Nam S, Li M, Choi K, Balch C, Kim S, Nephew KP: MicroRNA and mRNA integrated analysis (MMIA): a web tool for examining biological functions of microRNA expression. Nucleic Acids Res 2009, 37 Web Server: W356-362.

11. Papadopoulos GL, Alexiou P, Maragkakis M, Reczko M, Hatzigeorgiou AG: DIANA-mirPath: Integrating human and mouse microRNAs in pathways. Bioinformatics 2009, 25(15):1991-1993.

12. Papadopoulos GL, Reczko M, Simossis VA, Sethupathy P, Hatzigeorgiou AG The database of experimentally supported targets: a functional update of TarBase. Nucleic Acids Res 2009, 37 Database: D155-158.

13. Bartel DP: MicroRNAs: target recognition and regulatory functions. Cell 2009, 136(2):215-233.

14. Taccioli C, Fabbri E, Visone R, Volinia S, Calin GA, Fong LY, Gambari R, Bottoni A, Acunzo M, Hagan J, et al: UCbase \& miRfunc: a database of ultraconserved sequences and microRNA function. Nucleic Acids Res 2009, 37 Database: D41-48.

15. Dennis G Jr, Sherman BT, Hosack DA, Yang J, Gao W, Lane HC, Lempicki RA: DAVID: Database for Annotation, Visualization, and Integrated Discovery. Genome Biol 2003, 4(5):P3. 
16. Shi B, Guo Y, Wang J, Gao W: Altered expression of microRNAs in the myocardium of rats with acute myocardial infarction. BMC Cardiovasc Disord 10(1):11.

17. van Rooij E, Sutherland $L B$, Thatcher JE, DiMaio JM, Naseem RH, Marshall WS, Hill JA, Olson EN: Dysregulation of microRNAs after myocardial infarction reveals a role of miR-29 in cardiac fibrosis. Proc Natl Acad Sci USA 2008, 105(35):13027-13032.

18. Tanzer A, Stadler PF: Molecular evolution of a microRNA cluster. J Mol Biol 2004, 339(2):327-335.

19. Yu J, Wang F, Yang GH, Wang FL, Ma YN, Du ZW, Zhang JW: Human microRNA clusters: genomic organization and expression profile in leukemia cell lines. Biochem Biophys Res Commun 2006, 349(1):59-68.

20. Abbott AL, Alvarez-Saavedra E, Miska EA, Lau NC, Bartel DP, Horvitz HR, Ambros V: The let-7 MicroRNA family members mir-48, mir-84, and mir241 function together to regulate developmental timing in Caenorhabditis elegans. Dev Cell 2005, 9(3):403-414

21. Korpal M, Lee ES, Hu G, Kang Y: The miR-200 family inhibits epithelialmesenchymal transition and cancer cell migration by direct targeting of E-cadherin transcriptional repressors ZEB1 and ZEB2. J Biol Chem 2008, 283(22):14910-14914.

22. Altuvia $Y$, Landgraf $P$, Lithwick $G$, Elefant $N$, Pfeffer $S$, Aravin A, Brownstein MJ, Tuschl T, Margalit H: Clustering and conservation patterns of human microRNAs. Nucleic Acids Res 2005, 33(8):2697-2706.

23. Baskerville S, Bartel DP: Microarray profiling of microRNAs reveals frequent coexpression with neighboring miRNAs and host genes. RNA 2005, 11(3):241-247.

24. Xiao C, Rajewsky K: MicroRNA control in the immune system: basic principles. Cell 2009, 136(1):26-36.

25. Rivals I, Personnaz L, Taing L, Potier MC: Enrichment or depletion of a GO category within a class of genes: which test? Bioinformatics 2007, 23(4):401-407.

doi:10.1186/1471-2105-11-419

Cite this article as: Lu et al:: TAM: A method for enrichment and

depletion analysis of a microRNA category in a list of microRNAs. BMC

Bioinformatics 2010 11:419.

\section{Submit your next manuscript to BioMed Central and take full advantage of:}

- Convenient online submission

- Thorough peer review

- No space constraints or color figure charges

- Immediate publication on acceptance

- Inclusion in PubMed, CAS, Scopus and Google Scholar

- Research which is freely available for redistribution 